\title{
The microRNA miR-19a-3p suppresses cell growth, migration, and invasion in multiple myeloma via the $\mathrm{Wnt} / \beta$-catenin pathway
}

\author{
Zhengxiao Wei ${ }^{1}$, Wang Wang ${ }^{2}$, Qingfeng $\mathrm{Li}^{1}$, Linfang $\mathrm{Du}^{2}$, Xuemei $\mathrm{He}^{3}$ \\ ${ }^{1}$ Department of Clinical Laboratory, Public Health Clinical Medical Center of Chengdu, Chengdu, China; ${ }^{2}$ College of Life Sciences, Sichuan \\ University, Chengdu, China; ${ }^{3}$ Medical Experimental Center, Affiliated Hospital of Southwest Medical University, Luzhou, China \\ Contributions: (I) Conception and design: Z Wei, L Du, X He; (II) Administrative support: Q Li, L Du, X He; (III) Provision of study materials or \\ patients: Z Wei, W Wang, L Du, X He; (IV) Collection and assembly of data: Z Wei, W Wang; (V) Data analysis and interpretation: All authors; (VI) \\ Manuscript writing: All authors; (VII) Final approval of manuscript: All authors. \\ Correspondence to: Linfang Du, PhD. College of Life Sciences, Sichuan University, Chengdu 610064, China. Email: dulinfang89@163.com; Xuemei \\ He, PhD. Medical Experiment Center, Affiliated Hospital of Southwest Medical University, Luzhou 646000, China. Email: 1681@163.com.
}

\begin{abstract}
Background: MicroRNAs have been suggested as potential regulators in the development of multiple myeloma (MM) through affecting the expression of their target genes. This study aimed to investigate the effects of miR-19a-3p in MM, and its underlying mechanisms in regulating cell proliferation and invasion.

Methods: Bone marrow samples from $25 \mathrm{MM}$ patients and 12 healthy donors were collected and miR-19a$3 \mathrm{p}$ and Wnt1 mRNA expression was assessed. The effects of miR-19a-3p on cell proliferation, migration, and invasion in U226 and RPMI-8226 MM cells were evaluated by miR-19a-3p overexpression. Luciferase assays were performed to explore the potential target genes. Knock down or overexpression of Wnt1 was used to explore the effects of miR-19a-3p on cell growth, migration, and invasion.

Results: The expression of miR-19a-3p was downregulated in MM and cell lines, while Wnt1 mRNA levels were increased. Overexpression of miR-19a-3p inhibited cell proliferation, migration, and invasion in U226 and RPMI-8226 cells. Additionally, western blot assays revealed that miR-19a-3p could suppress Wnt1, $\beta$-catenin, cyclin D1, and c-Myc expression. Knockdown of Wnt1 also inhibited cell growth, migration, and invasion. Moreover, luciferase reporter assay revealed direct binding between Wnt1 and miR19a-3p. Wnt1 overexpression partially reversed the suppressive effects of miR-19a-3p on cell proliferation, migration, and invasion in U266 cells.
\end{abstract}

Conclusions: The expression of miR-19a-3p was downregulated in MM patients and MM cell lines. Overexpression of miR-19a-3p inhibited proliferation, migration, and invasion by targeting Wnt 1 via the Wnt/ $\beta$-catenin signaling pathway.

Keywords: miR-19a-3p; multiple myeloma (MM); proliferation; invasion; migration; Wnt1/ $\beta$-catenin pathway

Submitted Dec 02, 2020. Accepted for publication Feb 04, 2021.

doi: $10.21037 /$ tcr-20-3490

View this article at: http://dx.doi.org/10.21037/tcr-20-3490

\section{Introduction}

Multiple myeloma (MM) is the second most common hematological malignancy $(1,2)$ characterized by the uncontrolled growth of monoclonal plasma cells in the bone marrow, resulting in hypercalcemia, bone disease, renal failure, anemia and other complications $(3,4)$. The latest treatments for MM include immunomodulatory drugs
(IMID), second-generation proteasome inhibitors (PI), antiCD38 monoclonal antibodies (MoAb), and chimeric antigen receptor T cell (CAR T cell) therapy (5-8). Although these therapies significantly improve the rate of survival in $M M$ patients, issues of relapse and refractory disease still require urgent attention (9). Therefore, it is necessary to explore the pathogenesis and diagnostic markers of MM to provide new directions for the treatment of MM. 
Table 1 Sequences for miR-19a-3p and Wnt1 siRNA

\begin{tabular}{ll}
\hline Name & Sequence (5'-3') \\
\hline miR-19a-3p mimic & Sense: CGAUCGAAUCUGACGUACCUGAU; antisense: UGUGCAAAUCUAUGCAAAACUGA \\
mimic NC & Sense: UCGUUAUGGCCAUGUCAUAACGG; antisense: UUGACGUCUCUAUGCAAGACAGA \\
Wnt1 siRNA & Sense: CGCUCUUGGACGACAGAACTT; antisense: UGACAUUUGCCUACCUGGGTT \\
siRNA NC & Sense: UUGGCCGGAAGCCUCAGCUTT; antisense: GAGUUGCACCCUCAAGGCATT \\
\hline
\end{tabular}

MicroRNAs (miRNAs) are small non-coding RNA with a length of 19-25 bases that regulate gene expression by degrading mRNA or inhibiting its translation (10). In MM, a variety of miRNAs (such as miR-21 and miR-221) are abnormally expressed or dysfunctional. In addition, the miRNAs in the tumor microenvironment regulate MM cell functions through gene/protein targets, signaling molecules, and pathways leading to the transfer of MM cells (11-13). It has been reported that the regulation of miRNAs (such as miR-15a, miR-16, and miR-34) in MM cells could weaken their functional interaction with the bone marrow microenvironment and produce significant anti-tumor activity $(10,14)$. Other studies have demonstrated that miR$19 \mathrm{a}-3 \mathrm{p}$ was abnormally expressed in MM, and promoted cell proliferation and inhibited cell apoptosis by degrading the F-box only protein 32 (FBXO32) mRNA (15). Therefore, miRNAs may be potential therapeutic targets for MM.

The $W n t / \beta$-catenin pathway is widely involved in cell proliferation, tumorigenesis, and metastasis (16-18). Wnt is a type of secreted glycoprotein that interacts with specific receptors on the cell surface and causes $\beta$-catenin to accumulate through a series of downstream protein phosphorylation and dephosphorylation processes $(19,20)$. $\beta$-catenin enters the nucleus and regulates gene expression (such as cyclin D1 and c-Myc), and promotes tumorigenesis (21). Therefore, inhibiting the activation of the $\mathrm{Wnt} / \beta$-catenin pathway plays an important role in antitumorigenesis.

Since it has been shown that miR-19a-3p is dysregulated in several types of cancer cells (including MM cells), this study hypothesized that miR-19a-3p may be involved in the progression of MM. This investigation explored the novel regulatory mechanisms of miR-19a-3p in MM, and provided new clues for the treatment of MM. We present the following article in accordance with the MDAR reporting checklist (available at http://dx.doi.org/10.21037/ tcr-20-3490).

\section{Methods}

\section{Patient sample collection}

Bone marrow samples from 25 patients with MM and 12 patients with non-hematological diseases were collected in the Affiliated Hospital of Southwest Medical University from May 2017 to May 2018. The collected samples were stored at $-80{ }^{\circ} \mathrm{C}$. This study was approved by the Research Ethics Committee of the Affiliated Hospital of Southwest Medical University, and all patients provided signed informed consent. The study was conducted in accordance with the Declaration of Helsinki (as revised in 2013).

\section{Cell culture and transfection}

The human MM cell lines U266 and RPMI-8226 were purchased from the American Type Culture Collection (Manassas, VA, USA). The cells were grown in Roswell Park Memorial Institute (RPMI)-1640 medium (Hyclone; Logan, UT, USA) supplemented with $10 \%$ fetal bovine serum (FBS; Gibco; Thermo Fisher Scientific, Inc.) and cultured at $37^{\circ} \mathrm{C}$ with $5 \% \mathrm{CO}_{2}$ atmosphere.

The small interfering RNA of Wnt1 (si-Wnt1), the miR19a-3p mimic, and the pcDNA3.1-Wnt1 vector, as well as their corresponding negative controls (NC) were obtained from Shanghai GenePharma Co., Ltd., (Shanghai, China) and transfected into U266 and RPMI-8226 cells using Lipofectamine 2000 (Invitrogen; Thermo Fisher Scientific, Inc.) following the manufacturer's protocol. The sequences of miR-19a-3p mimic and si-Wnt1 were listed in Table 1 . Cell samples were then harvested for further research.

\section{Cell counting kit-8 (CCK-8) assay}

Cells at the logarithmic growth phase were harvested and digested with trypsin to prepare a cell suspension. The cells were seeded into 96-well plates at a cell density of $2 \times 10^{3}$ cells/ 
Table 2 The sequence of primers used in this study

\begin{tabular}{ll}
\hline Name & Sequence (5'-3') \\
\hline miR-19a-3p & Sense: GCGTGTGCAAATCTATGCAA; antisense: AGTGCAGGGTCCGAGGTATT \\
Wnt1 & Sense: CTCATGTGATCTATGCCCGTC; antisense: AGGTGATACAACTCGTTCGTAGT \\
U6 & Sense: CTCGCTTCGGCAGCACA; antisense: AACGCTTCACGAATTTGCGT \\
GAPDH & Sense: AGAAGGCTGGGGCTCATTT; antisense: AGGGGCCATCCACAGTCTTC \\
\hline
\end{tabular}

well, and the wells at the edge of the plate were filled with sterile phosphate buffered saline (PBS). After cell culture for $0,24,36,48$, and 60 hours, $10 \mu \mathrm{L}$ of the CCK- 8 reagent was added to each well and cultured for a further 4 hours. A microplate reader was used to measure absorption at $450 \mathrm{~nm}$.

\section{Flow cytometry analysis}

U226 cells were transfected with miR-19a-3p mimic and mimic NC and cultured for 24 hours. After trypsin digestion, cell suspension at a cell density of $1 \times 10^{6}$ cells $/ \mathrm{mL}$ was made by $1 \times$ Banding Buffer dilution. Then cell suspension was added $5 \mu \mathrm{L}$ Annexin V-FITC and $10 \mu \mathrm{L}$ PI, and incubate for $15 \mathrm{~min}$ at room temperature in the dark. Finally, flow cytometry was used to analyze apoptosis of each sample group.

\section{Wound bealing analysis}

The transfected cells were seeded into 24-well cell culture plates at a density of $5 \times 10^{4}$ cells/well. After 24 hours, the cell confluence reached $70-80 \%$. A sterile $10 \mu \mathrm{L}$ pipette tip was used to gently draw a straight line through the monolayer of cultured cells. After scratching, each well was gently washed with PBS to remove the exfoliated cells. Cells were cultured for a further 48 hours and wound closure was examined under light microscopy.

\section{Transwell invasion assay}

A 24-well transwell chamber (Corning Inc., Corning, NY, USA) was pre-coated with Matrigel (BD Biosciences, USA). The transfected cells were resuspended with serum-free Dulbecco's Modified Eagle Medium (DMEM) and then seeded into the upper chamber at a density of $5 \times 10^{4}$ cells/ well. DMEM containing with $10 \%$ FBS was added into the lower chamber. After 48 hours at $37^{\circ} \mathrm{C}$, the cells on the lower surface were fixed with $4 \%$ methanol for 10 minutes and stained with $0.1 \%$ crystal violet for 5 minutes. Invasive cells were observed in six randomly selected fields of view under a light microscope.

\section{Quantitative reverse transcription polymerase chain reaction ( $R T-q P C R)$}

Total RNA was extracted from clinical samples and transfected cell lines using TRIzol reagent (Invitrogen; Thermo Fisher Scientific, Inc.) according to the manufacturer's instructions. To obtain the cDNA from miRNA, the miRNA 1st Strand cDNA Synthesis Kit (by stem-loop) (Vazyme Biotech Co., Ltd) was used to reverse transcribe miR-19a-3p according to the product instructions. For mRNA, template RNA was reverse transcribed to obtain cDNA using Hiscript ${ }^{\circledR}$ II Q RT SuperMix (Vazyme Biotech Co., Ltd) following the manufacturer's protocols. RT-qPCR was performed using AceQ $^{\circledR}$ qPCR SYBR Green Master Mix (Vazyme Biotech Co., Ltd). The following qPCR program was used: predenaturation at $95{ }^{\circ} \mathrm{C}$ for 10 minutes; 40 cycles of $95{ }^{\circ} \mathrm{C}$ for 10 seconds, $55^{\circ} \mathrm{C}$ for 10 seconds, $72{ }^{\circ} \mathrm{C}$ for 20 seconds. The relative miR-19a-3p and Wnt 1 mRNA expression levels were calculated using the $2^{-\Delta \Delta \mathrm{Ct}}$ method. The primer sequences used in this study are shown in Table 2.

\section{Western blot analysis}

Total protein was isolated from the MM tissues and cells using RIPA lysis buffer (Thermo Scientific, Waltham, MA, USA). The Pierce ${ }^{\mathrm{TM}}$ BCA Protein Assay Kit (Thermo Scientific, Waltham, MA, USA) was used to measure protein concentration. Equal amounts of protein $(40 \mu \mathrm{g})$ for each sample were separated by $10 \%$ SDS-PAGE (sodium dodecyl sulphate-polyacrylamide gel electrophoresis) at a constant voltage $(120 \mathrm{~V})$ and then transferred onto polyvinylidene fluoride (PVDF) membranes at a constant current (200 mA). After blocking with $5 \%$ skimmed milk at room temperature for 1 hour, the membranes were incubated with primary antibodies (Wnt1 \#ab15251 dilution 1:1,000, Abcam. 
$\beta$-catenin \#8480, dilution 1:1,000; Cyclin D1 \#55506, dilution 1:1,000; c-Myc \#5605, dilution 1:1,000; $\beta$-actin \#4970, dilution 1:1,000, Cell Signaling Technology, Inc.) overnight at $4{ }^{\circ} \mathrm{C}$. Membranes were washed with tris buffered saline tween (TBST) and incubated with secondary antibodies (anti-rabbit IgG, HRP-linked antibody \#7074, dilution 1:2,000, Cell Signaling Technology, Inc.) for 1 hour at room temperature and visualized using enhanced chemiluminescence.

\section{Dual-luciferase reporter assay}

The online miRNA target predication databases miRDB and TargetScan 7.2 predicted that the target gene of miR19a-3p was Wnt1. The wild-type (WT) and mutant-type (Mut) of the 3'UTR of Wnt1 was cloned and amplified by PCR, and the psi-CHECK2 ${ }^{\mathrm{TM}}$ plasmids containing the wild-type and mutant-type fragments of the 3'-UTR of Wnt1 were constructed. Cells were cotransfected with the miR-19a-3p mimic, the corresponding negative control (NC) mimic, or the Wnt1 WT, or Mut luciferase reporter vector using Lipofectamine 2000 reagent according to the instruction manual. After transfection for 48 hours, the Dual-Luciferase Reporter Assay kit (Promega Corporation) was used to detect Firefly and Renilla luciferase activities following the manufacturer's protocol.

\section{Statistical analysis}

GraphPad Prism 6.0 software (GraphPad, Inc.) was used to conduct data analysis. Data were expressed as the means \pm standard deviation (SD). Student's $t$-test and one-way of variance analysis (ANOVA) was used for comparisons between multiple groups. A P value $<0.05$ was considered statistically significant.

\section{Results}

The expression of miR-19a-3p was decreased, while the expression of Wnt1 was upregulated in MM tissues and cell lines

To investigate the expression of miR-19a-3p and Wnt 1 in MM samples and cells, RT-qPCR and western blot analysis were performed. The expression of miR-19a$3 \mathrm{p}$ was significantly reduced in the bone marrow samples from patients with $\mathrm{MM}$ compared to samples from healthy donors (Figure 1A). The expression of Wnt1 mRNA was increased in MM patients (Figure $1 B$ ). Western blot detection demonstrated that the expression of the Wnt1 protein was consistent with the mRNA changes (Figure $1 C)$. In addition, the expression of miR-19a-3p and Wnt1 was studied in two human MM cell lines (U226 and RPMI8226). The results showed that miR-19a-3p was downregulated in both U226 and RPMI-8226 cells compared to healthy donor cells (Figure 1D). At the same time, Wnt1 mRNA and protein levels increased significantly in both U226 and RPMI-8226 cells (Figure 1E,F).

\section{Overexpression of miR-19a-3p suppressed cell proliferation in MM cell lines}

The miR-19a-3p overexpression model was established in U226 and RPMI-8226 cells, and RT-qPCR was used to detect the transfection efficiency of the miR-19a$3 \mathrm{p}$ mimic. As illustrated in Figure $2 A$, the level of miR$19 a-3 p$ expression was greatly increased in U226 and RPMI-8226 cells after transfection with the miR-19a$3 \mathrm{p}$ mimic compared to the NC mimic. The CCK-8 assay was performed to assess the effects of miR-19a-3p overexpression on cell proliferation. The ability of U226 and RPMI-8226 cells to proliferate was inhibited following transfection with the miR-19a-3p mimic (Figure 2B,C). Flow cytometry analysis was performed to assess the effect of miR-19a-3p on the apoptosis of U226 cells. The result discovered that miR-19a-3p mimic promoted cell apoptosis on U226 cells compared to mimic NC group (Figure 2D,E).

\section{Overexpression of miR-19a-3p inbibited cell migration and invasion in MM cell lines}

To explore the effects of miR-19a-3p on cell migration and invasion, wound healing analysis and transwell invasion experiments were performed on U226 and RPMI-8226 cells. The ability of U226 cells to migrate decreased following transfection with the miR-19a-3p mimic, compared with the NC mimic (Figure 3A). Quantitative analysis of wound closure further verified that overexpression of miR-19a-3p suppressed U226 cell migration (Figure 3B). Cell migration ability in RPMI-8226 cells was similarly greatly inhibited following transfection with the miR-19a-3p mimic (Figure $3 C, D)$. In addition, miR-19a-3p overexpression significantly depressed the ability of both U226 and RPMI-8226 cells to invade into the Matrigel as shown by the transwell experiment (Figure 3E). Quantitative assessment of the number of invasive cells also demonstrated that the invasion capability of U226 and RPMI-8226 cells were blocked by 
A
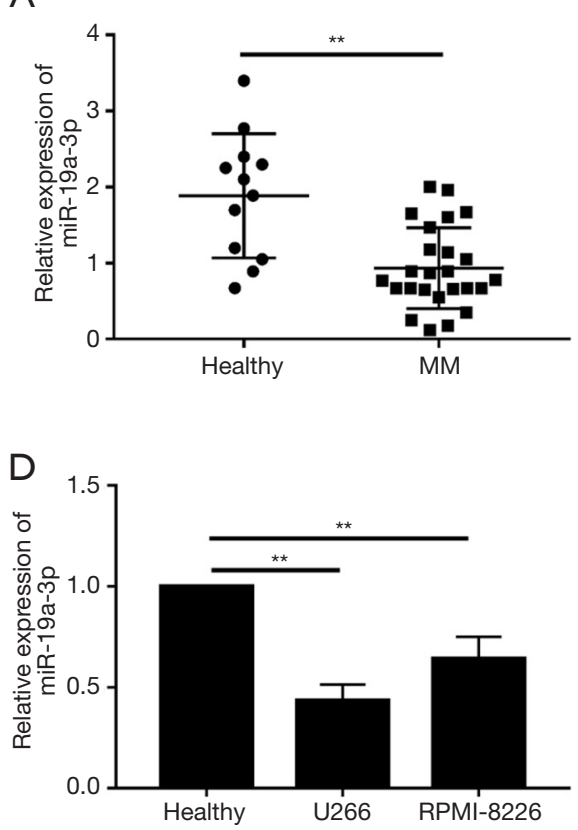

B

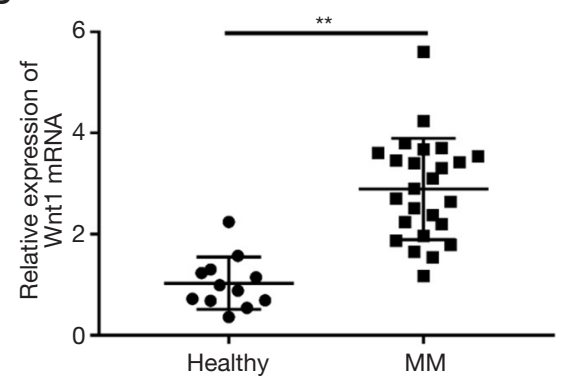

$\mathrm{E}$

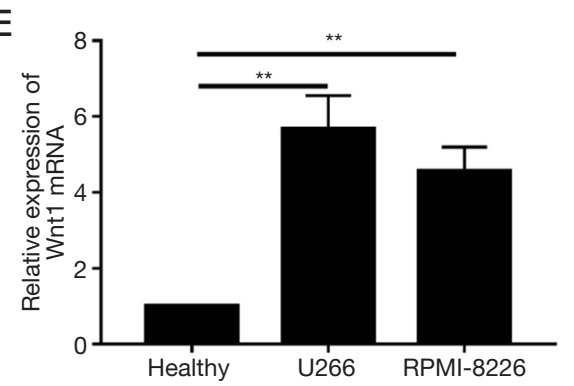

C

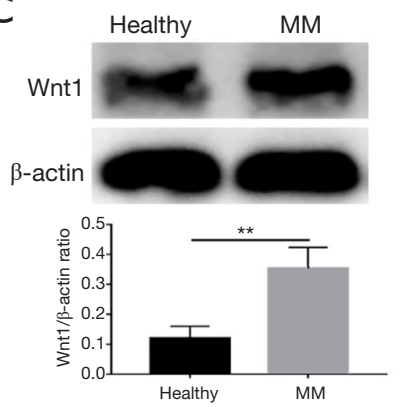

$\mathrm{F}$

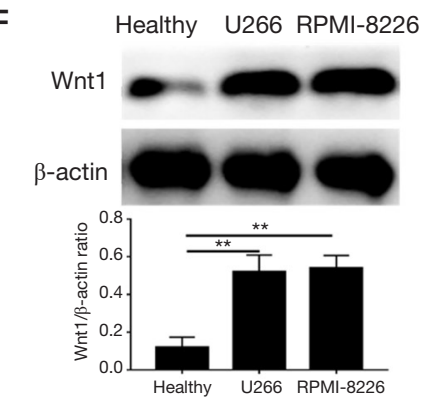

Figure 1 The expression of miR-19a-3p was decreased, while expression of Wnt1 was upregulated in multiple myeloma tissues and cell lines. (A) RT-qPCR analysis of miR-19a-3p expression in the bone marrow samples from patients with MM and healthy donors. (B) The levels of Wnt1 mRNA in MM patients and healthy donors were detected by RT-qPCR. (C) Western blot analysis was used to examine Wnt1 protein expression in MM patients and healthy donors. (D) The expression of miR-19a-3p in MM cell lines (U226 and RPMI-8226) compared with normal donor samples was determined using RT-qPCR. (E) The expression of Wnt1 mRNA in U226 and RPMI-8226 cells was detected by RT-qPCR. (F) Wnt1 protein levels in U226 and RPMI-8226 cells were measured by western blot analysis. ${ }^{*} \mathrm{P}<0.01$. RTqPCR, quantitative reverse transcription polymerase chain reaction; $M M$, multiple myeloma.

miR-19a-3p overexpression (Figure 3F). The above data suggested that miR-19a-3p overexpression inhibited the cell migration and invasion ability of MM cells.

\section{Wnt1 was a direct target of $m i R-19 a-3 p$}

To further explore the mechanisms by which miR-19a$3 p$ affects the progression of $M M$, the Wnt $1 / \beta$-catenin pathway was analyzed using U226 and RPMI-8226 cells. The results demonstrated that overexpression of miR-19a$3 \mathrm{p}$ significantly suppressed Wnt1 mRNA expression in both U226 and RPMI-8226 cells (Figure 4A). As presented in Figure 4B, Western blot analysis detected the proteins involved in the $\mathrm{Wnt} 1 / \beta$-catenin pathway. Wnt1, $\beta$-catenin, cyclin D1, and c-Myc were all significantly inhibited in the miR-19a-3p mimic group compared to the NC mimic group in both U226 and RPMI-8226 cells. Furthermore, analyses using the Targetscan and miRBD databases predicted that miR-19a-3p and Wnt1 have complementary base pairing sequences (Figure 4C). In addition, the interaction between miR-19a-3p and Wnt1 was verified by dual luciferase reporter analysis. The results demonstrated that miR-19a-3p targeted and negatively regulated Wnt1 mRNA expression in both U226 and RPMI-8226 cells (Figure 4D,E). These data indicated that miR-19a-3p targeted Wnt1 and affected the activation of the $\mathrm{Wnt} 1 / \beta$-catenin pathway.

\section{Silencing of Wnt1 suppressed cell proliferation, migration, and invasion in U266 cells}

To determine whether silencing of Wnt1 had the same effect as miR-19a-3p overexpression, siRNA was used. The silencing efficiency of si-Wnt1 was assessed by RTqPCR and Western blot analysis. The results demonstrated that the expression of Wnt 1 mRNA and protein was significantly suppressed after transfection with si-Wnt1 (Figure 5A,B). Knockdown of Wnt1 greatly inhibited U226 cell proliferation compared to the si-NC (Figure 5C). The 
A

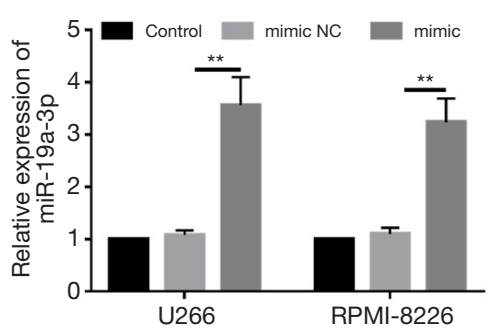

D

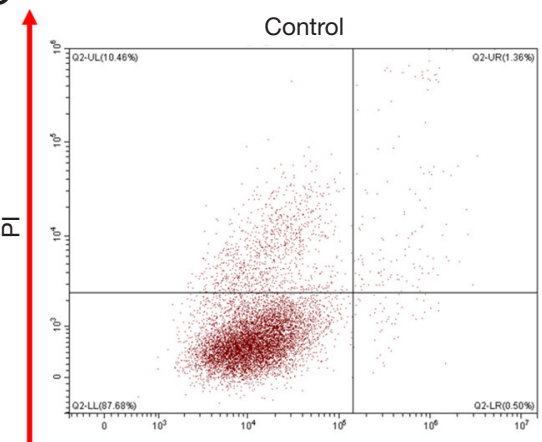

B
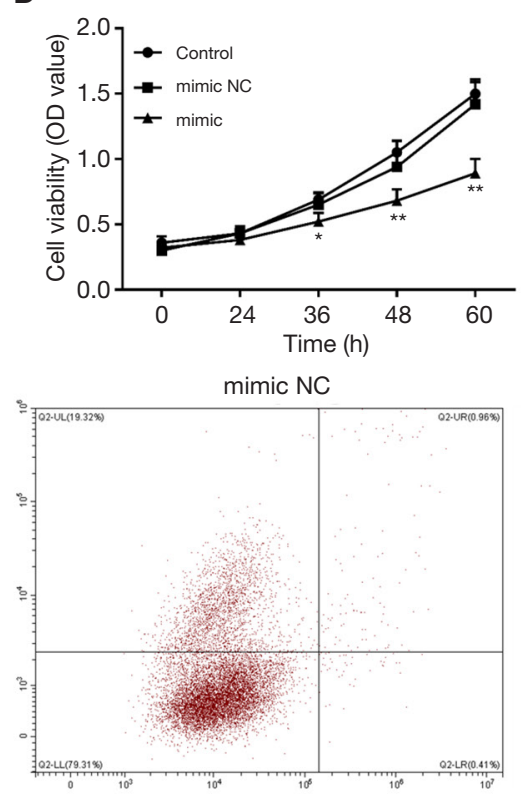

Annexin V-FITC
C RPMI-8226
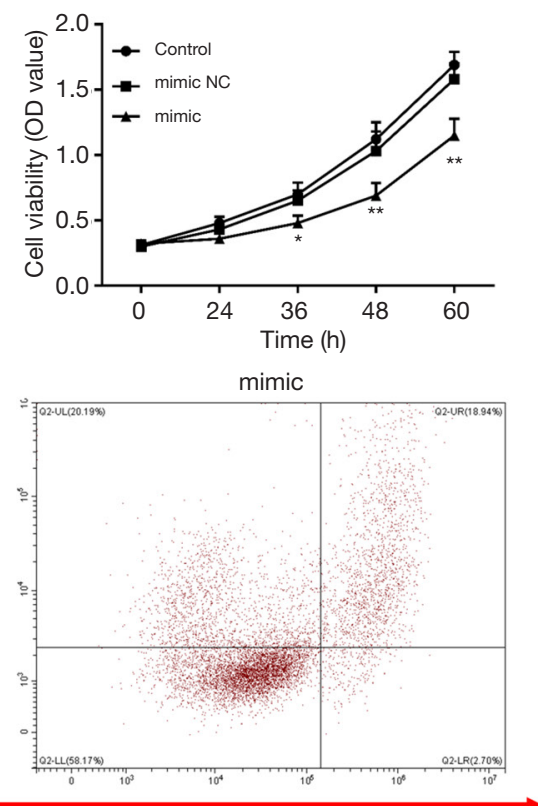

$E$

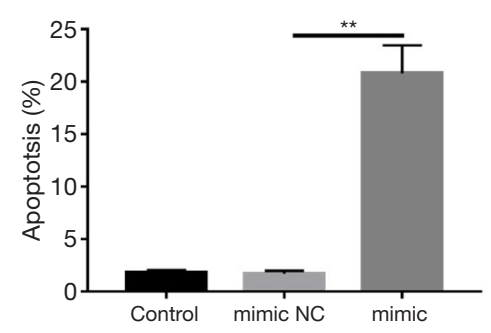

Figure 2 Overexpression of miR-19a-3p suppressed cell proliferation in multiple myeloma cell lines. U226 and RPMI-8226 cells were transfected with a miR-19a-3p mimic or a NC mimic. (A) RT-qPCR analysis of miR-19a-3p levels in U226 and RPMI-8226 cells. (B) Cellular growth curves for U226 cells as measured by the CCK-8 assay. (C) Cellular growth curves for RPMI-8226 cells as measured by the CCK-8 assay. (D,E) Apoptosis was detected by flow cytometry on U226 cells. ${ }^{*} \mathrm{P}<0.05$; ${ }^{* *} \mathrm{P}<0.01$. NC, negative control; RT-qPCR, quantitative reverse transcription polymerase chain reaction; CCK-8, cell counting kit-8; PI, propidium Iodide; FITC, fluoresceine isothiocyanate.

wound closure speed was also attenuated by silencing $\mathrm{Wnt} 1$ in U226 cells (Figure 5D). Moreover, the number of invasive cells was similarly reduced by silencing Wnt1 in U226 cells (Figure 5E). The above results indicated that silencing Wnt1 had the same effect as miR-19a-3p overexpression on the proliferation, migration, and invasion of U226 cells.

\section{Wnt1 overexpression partially reversed the suppressive effects of miR-19a-3p on cell proliferation, migration, and invasion in U266 cells}

Wnt1 is one of the key proteins that affect the proliferation, migration, and invasion of MM cells. Therefore, the effects of Wnt1 overexpression on the miR-19a-3pmediated suppression of cell proliferation, migration, and invasion were assessed in U266 cells. The overexpression efficiency of Wnt1 was examined by RT-qPCR, and the results revealed that the levels of Wnt1 mRNA increased significantly after transfection of the pcDNA3.1-Wnt1 plasmid (Figure 6A). Western blot analysis was performed to explore the effects of Wnt1 overexpression on the Wnt1/ $\beta$-catenin pathway. As shown in Figure $6 B$, the levels of Wnt1, $\beta$-catenin, cyclin D1, and c-Myc in cells transfected with the miR-19a-3p mimic and pcDNA3.1-Wnt1 
A

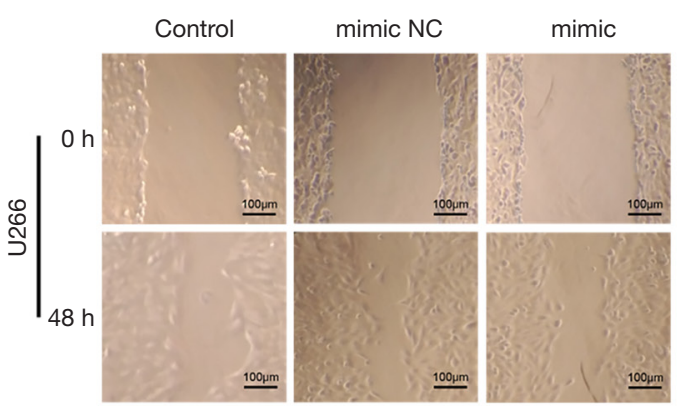

C

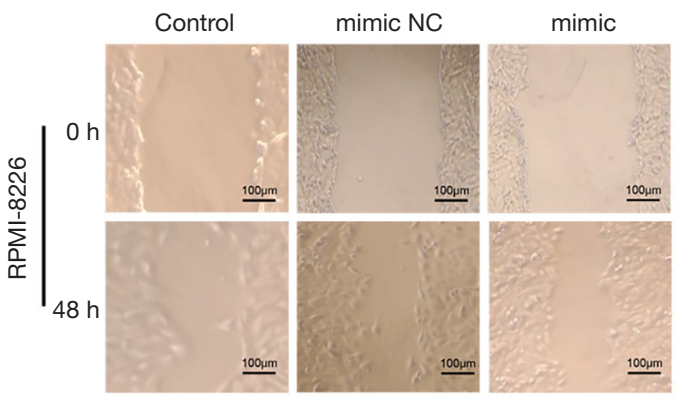

$\mathrm{E}$

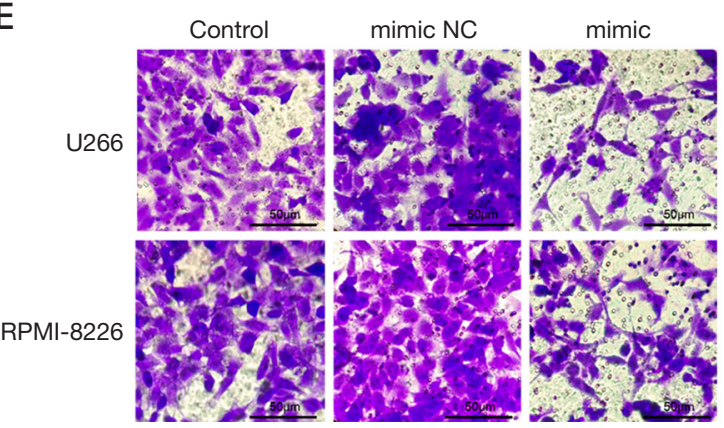

B

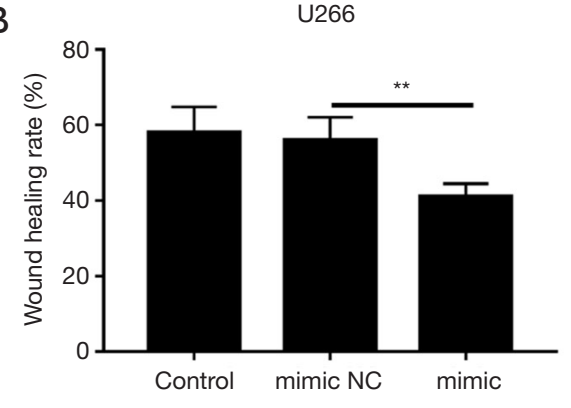

RPMI-8226
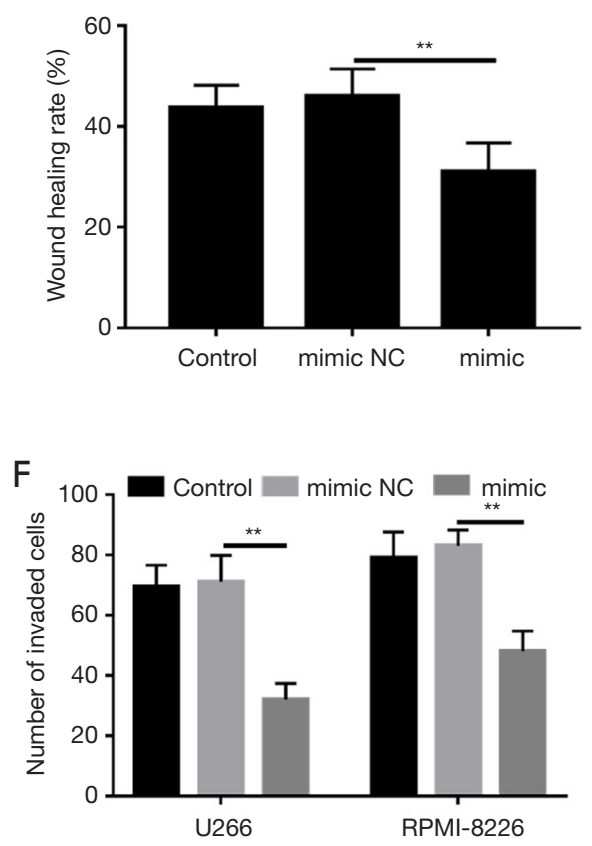

Figure 3 Overexpression of miR-19a-3p inhibited cell migration and invasion in multiple myeloma cell lines. U226 and RPMI-8226 cells were transfected with a miR-19a-3p mimic or a NC mimic. (A,C) The effect of miR-19a-3p overexpression on the migratory ability of U226 and RPMI-8226 cells was evaluated by the wound healing assay (magnification, 100x). (B,D) The wound closure rate was calculated with Image J software. (E) The effect of miR-19a-3p overexpression on the ability of U226 and RPMI-8226 cells to invade was assessed by the transwell invasion assay. The cells in the lower layer of the chamber were fixed with $4 \%$ paraformaldehyde for 20 minutes and stained with crystal violet solution for 15 minutes, and finally photographed under a microscope (magnification, 400x). (F) The number of invading cells was counted. ${ }^{*} \mathrm{P}<0.01$. NC, negative control.

(mimic+pcDNA3.1-Wnt1) were significantly higher than that in cells transfected with the miR-19a-3p mimic and pcDNA3.1 (mimic+pcDNA3.1) (Figure 6B). In addition, the proliferation, migration, and invasion capabilities of U226 cells were analyzed. The CCK-8 assay results revealed that overexpression of Wnt 1 significantly improved the viability of U226 cells overexpressing miR-19a-3p (Figure 6C). Wound healing analysis also demonstrated that the cell migration ability was significantly enhanced in the mimic+pcDNA3.1-Wnt1 group compared to the mimic+pcDNA3.1 group (Figure 6D). Transwell invasion analysis suggested that overexpression of Wnt1 reversed the inhibitory effects of miR-19a-3p on cell invasion (Figure 6E). These data indicated that Wnt1 overexpression partially reversed the suppressive effects of miR-19a-3p on cell proliferation, migration, and invasion in U266 cells. 

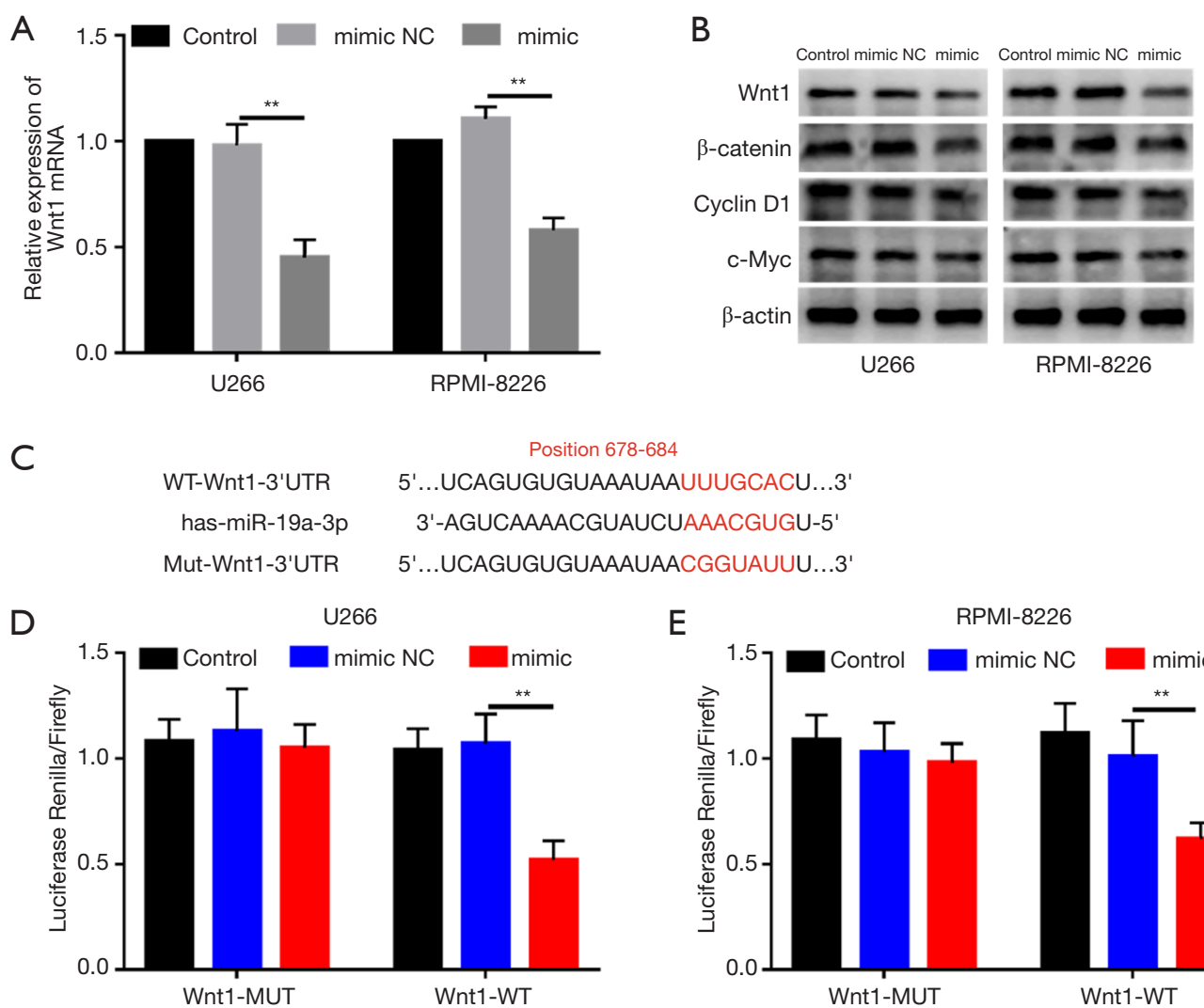

sition 678-684
GGUAAAUAAUUUGCACU...3'
AACGUAUCUAAACGUGU-5'
GGUAAUAACGGUAUUU...3'

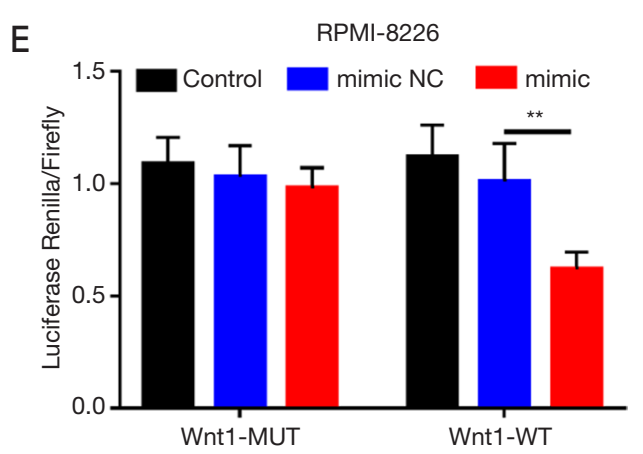

Figure 4 Wnt1 was a direct target of miR-19a-3p. U226 and RPMI-8226 cells were transfected with a miR-19a-3p mimic or a NC mimic. (A) The expression of Wnt1 mRNA in U226 and RPMI-8226 cells was detected using RT-qPCR. (B) Western blot analysis was performed to detect Wnt1, $\beta$-catenin, cyclin D1, and c-Myc. $\beta$-actin served as internal reference. (C) The sequence of miR-19a-3p revealed complementary pairing with the 3'-UTR of Wnt1. The sequence of the mutant 3'-UTR of Wnt1 is also shown. (D,E) The dual-luciferase reporter assay was performed on both U226 and RPMI-8226 cells. ${ }^{* *} \mathrm{P}<0.01$. RT-qPCR, quantitative reverse transcription polymerase chain reaction; NC, negative control.

\section{Discussion}

MM is one of the most common hematological malignancies, accounting for about $1 \%$ of all cancer cases (22). Currently, there is no drug or treatment that can completely cure MM. Resistance and relapse are the biggest obstacles hindering the development of successful MM treatments (8). Therefore, it is crucial to investigate the potential pathogenic mechanisms of the occurrence and development of MM, especially in terms of proliferation and apoptosis in order to develop new treatment strategies (1). In recent years, the use of non-coding RNAs (including miRNAs, long non-coding RNAs, circular RNAs, and siRNA) has become increasingly popular for studying the mechanisms and treatments of MM $(23,24)$. This present study demonstrated that miR-19a-3p was significantly down- regulated in MM patients and cell lines. This result was consistent with the changes in $\mathrm{miR}-19 \mathrm{a}-3 \mathrm{p}$ reported in other cancers $(15,25)$.

Furthermore, the mechanisms of miR-19a-3p were explored in MM cells. Our results revealed that miR-19a$3 \mathrm{p}$ inhibited cell proliferation, migration, and invasion in U226 and RPMI-8226 cells. This agreed with previous reports showing that overexpression of miRNA-19a-3p significantly reduced the cell proliferation, migration, and invasion ability of HCT116 human colon cancer cells (26). To investigate the mechanisms of miRNA-19a-3p-mediated inhibition of cell proliferation, migration, and invasion, bioinformatics software was used to predict the target genes of miRNA-19a-3p. The results identified that miRNA19a-3p binds to the 3'-UTR of Wnt1. Moreover, the dual luciferase report assay confirmed that microRNA-19a-3p 

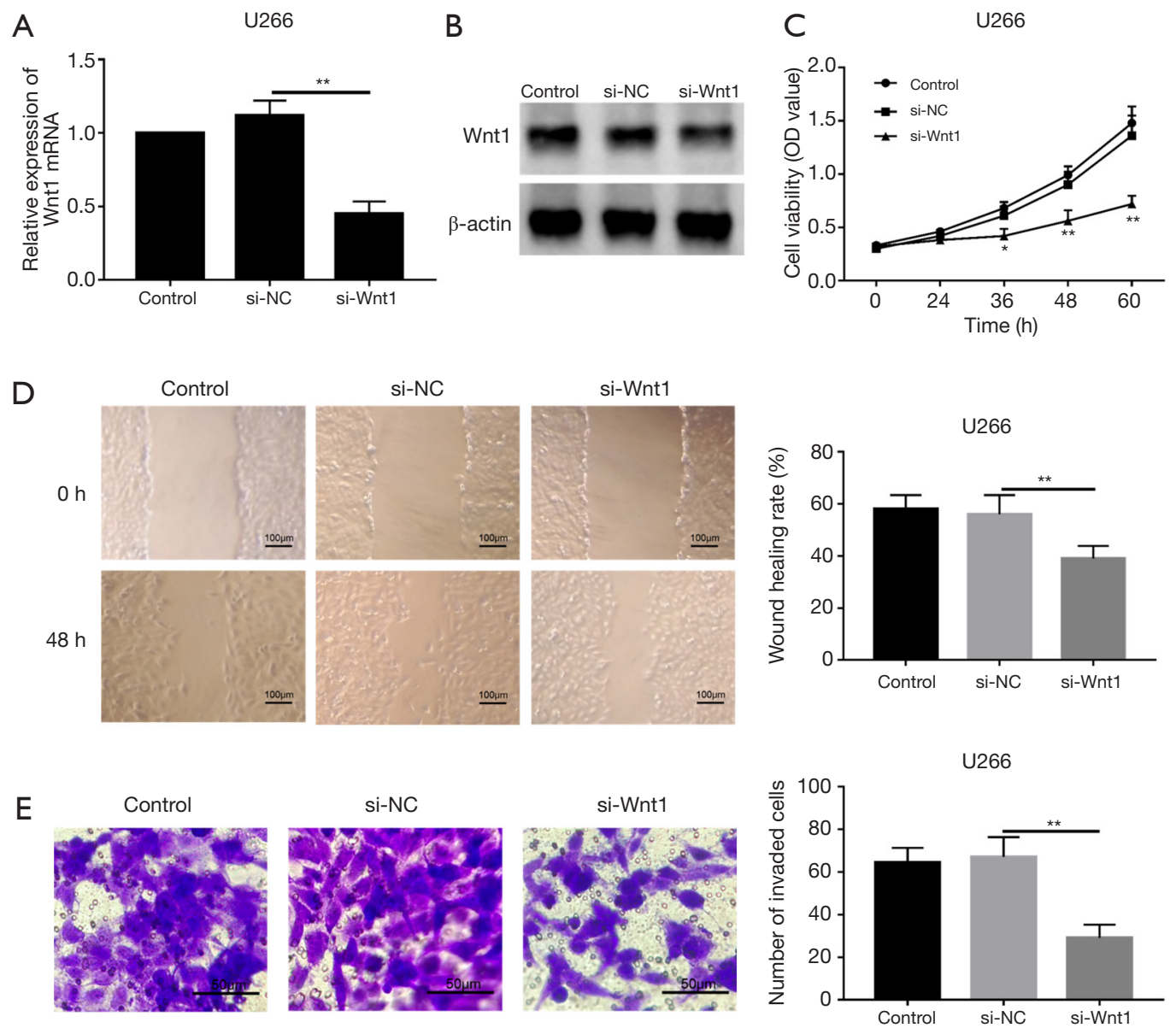

Figure 5 Knockdown of Wnt1 suppressed cell proliferation, migration, and invasion in U266 cell. U226 cells were transfected with 40 nM si-Wnt1 and its negative control si-NC. (A) The mRNA levels of Wnt1 were assessed using RT-qPCR. (B) Western blot was performed to detect Wnt1 protein expression after silencing Wnt1. (C) Cell viability was determined using the CCK-8 kit. (D) Wound healing assay was performed to determine the effect of si-Wnt on the migratory ability of U226 cells (magnification, 100x). (E) Transwell invasion assays were used to detect the invasion ability of U226 cells following knockdown of Wnt1. The cells in the lower layer of the chamber were fixed with 4\% paraformaldehyde for 20 minutes and stained with crystal violet solution for 15 minutes, and finally photographed under a microscope (magnification, $400 \times$ ). ${ }^{*} \mathrm{P}<0.05 ;{ }^{*} \mathrm{P}<0.01$. NC, negative control; RT-qPCR, quantitative reverse transcription polymerase chain reaction; CCK-8, cell counting kit-8.

negatively regulated the expression of $W n t 1$. Subsequently, Wnt1 knockdown and overexpression experiments demonstrated that miRNA-19a-3p inhibited cell proliferation, migration, and invasion by targeting $\mathrm{Wnt} 1$.

The $W n t / \beta$-catenin pathway plays an important role in embryogenesis, cell growth, and proliferation (27). Overactivation of the Wnt signaling pathway is inextricably linked to cancer initiation and progression (28). Wnt binds to the receptor and initiates a signal cascade to activate $\beta$-catenin which promotes the expression of oncogenes such as cyclin D1 and c-Myc (29). In this study, miR-19a-
$3 \mathrm{p}$ significantly inhibited the expression of $W \mathrm{nt} 1, \beta$-catenin, cyclin D1, and c-Myc. When Wnt1 was knocked down, the expression of $\beta$-catenin, cyclin D1, and c-Myc was also suppressed. These data suggested that Wnt1 played a vital role in the miR-19a-3p-mediated regulation of cell proliferation, migration, and invasion.

\section{Conclusions}

In summary, miR-19a-3p was down-regulated in MM tissues and cell lines and was negatively correlated with 

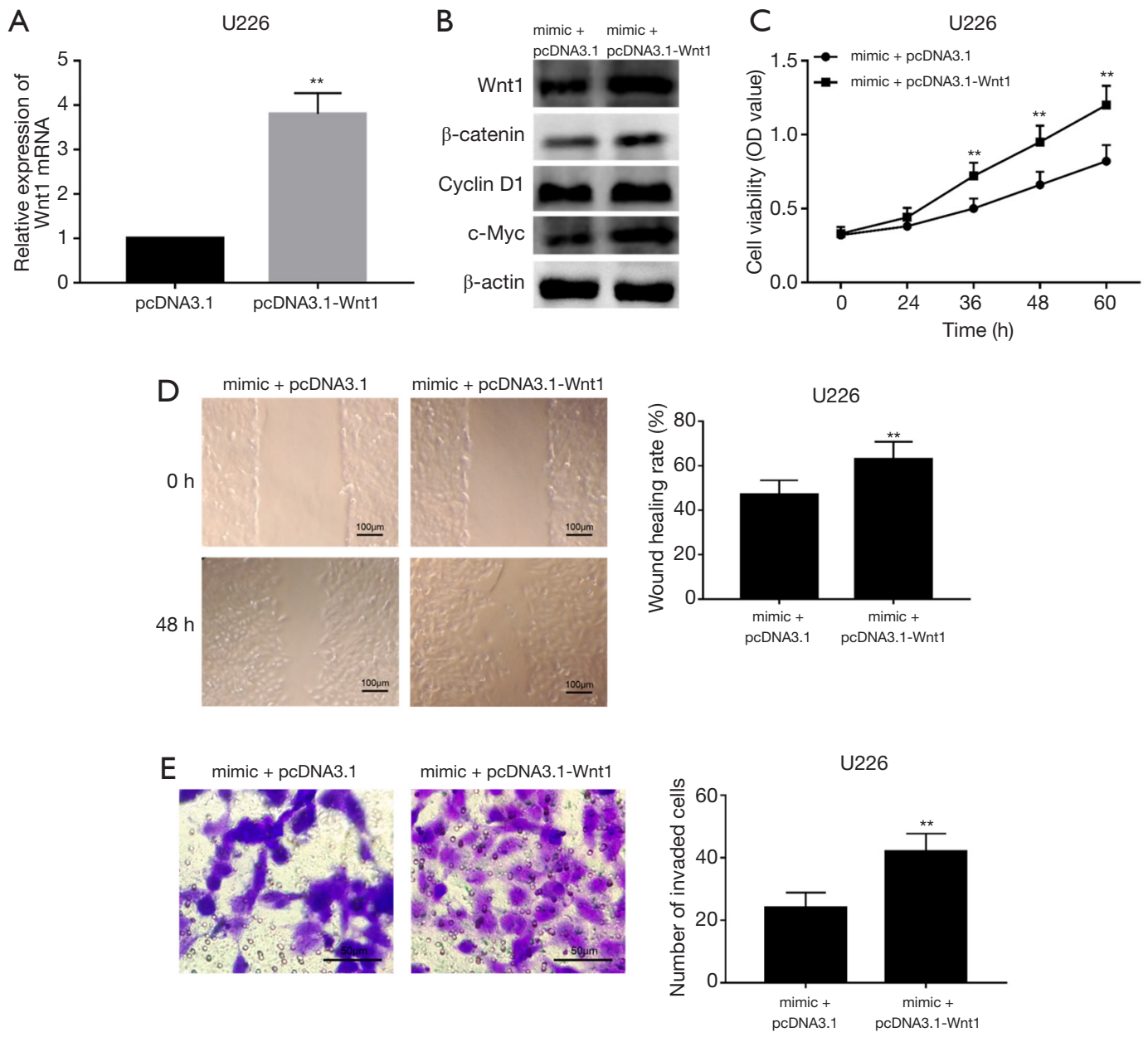

Figure $6 \mathrm{Wnt} 1$ overexpression partially reversed the suppressive effects of miR-19a-3p on cell proliferation, migration, and invasion in U266 cells. U266 cells were transfected with the pcDNA3.1 or pcDNA3.1-Wnt1 vector. (A) The mRNA levels of Wnt1 were measured to detect the transfection efficiency using RT-qPCR. (B) Western blot analysis was performed to determine Wnt1, $\beta$-catenin, cyclin D1, and c-Myc protein levels following Wnt1 overexpression. $\beta$-actin served as internal reference. (C) The CCK- 8 assay was used to assess cell viability. (D) Cell migration ability was evaluated by wound healing experiments (magnification, 100x). (E) Cell invasion ability was assessed using the transwell invasion assay. The cells in the lower layer of the chamber were fixed with $4 \%$ paraformaldehyde for 20 minutes and stained with crystal violet solution for 15 minutes, and finally photographed under a microscope (magnification, 400×). ** $<<0.01$. RT-qPCR, quantitative reverse transcription polymerase chain reaction; CCK-8, cell counting kit-8.

Wnt1 expression. Moreover, miR-19a-3p overexpression in U226 and RPMI-8226 cells inhibited cell growth, invasion, and migration by targeting $\mathrm{Wnt} 1$. The data in this report indicated that miR-19a-3p may be a potential target for the treatment of MM.

\section{Acknowledgments}

Funding: None.

\section{Footnote}

Reporting Checklist: The authors have completed the MDAR reporting checklist. Available at http://dx.doi.org/10.21037/ tcr-20-3490

Data Sharing Statement: Available at http://dx.doi. org/10.21037/tcr-20-3490

Conflicts of Interest: All authors have completed the ICMJE 
uniform disclosure form (available at http://dx.doi. org/10.21037/tcr-20-3490). The authors have no conflicts of interest to declare.

Ethical Statement: The authors are accountable for all aspects of the work in ensuring that questions related to the accuracy or integrity of any part of the work are appropriately investigated and resolved. This study was approved by the Research Ethics Committee of the Affiliated Hospital of Southwest Medical University, and all patients provided signed informed consent. The study was conducted in accordance with the Declaration of Helsinki (as revised in 2013).

Open Access Statement: This is an Open Access article distributed in accordance with the Creative Commons Attribution-NonCommercial-NoDerivs 4.0 International License (CC BY-NC-ND 4.0), which permits the noncommercial replication and distribution of the article with the strict proviso that no changes or edits are made and the original work is properly cited (including links to both the formal publication through the relevant DOI and the license). See: https://creativecommons.org/licenses/by-nc-nd/4.0/.

\section{References}

1. Zhong L, Xu Z, Jin X, et al. miR-451a suppression of IL6R can inhibit proliferation and increase apoptosis through the JAK2/STAT3 pathway in multiple myeloma. Oncol Lett 2020;20:339.

2. Fais $\mathrm{S}$, Marunaka $\mathrm{Y}$. The Acidic Microenvironment: Is It a Phenotype of All Cancers? A Focus on Multiple Myeloma and Some Analogies with Diabetes Mellitus. Cancers (Basel) 2020;12:3226.

3. Tian F, Wang H, Ma H, et al. miR-144-3p inhibits the proliferation, migration and angiogenesis of multiple myeloma cells by targeting myocyte enhancer factor $2 \mathrm{~A}$. Int J Mol Med 2020;46:1155-65.

4. Brigle K, Rogers B. Pathobiology and Diagnosis of Multiple Myeloma. Semin Oncol Nurs 2017;33:225-36.

5. Rodríguez-Otero P, Prósper F, Alfonso A, et al. CAR T-Cells in Multiple Myeloma Are Ready for Prime Time. J Clin Med 2020;9:3577.

6. Kumar SK, Rajkumar SV, Dispenzieri A, et al. Improved survival in multiple myeloma and the impact of novel therapies. Blood 2008;111:2516-20.

7. Gandhi UH, Cornell RF, Lakshman A, et al. Outcomes of patients with multiple myeloma refractory to CD38- targeted monoclonal antibody therapy. Leukemia 2019; 33:2266-75.

8. Devarakonda S, Cottini F, Bumma N, et al. Multiple Myeloma: Clinical Updates from the American Society of Clinical Oncology Annual Scientific Symposium 2020. J Clin Med 2020;9:3626.

9. Yang $\mathrm{Y}, \mathrm{Li} \mathrm{Y}, \mathrm{Gu} \mathrm{H}$, et al. Emerging agents and regimens for multiple myeloma. J Hematol Oncol 2020;13:150.

10. Handa $\mathrm{H}$, Murakami $Y$, Ishihara R, et al. The Role and Function of microRNA in the Pathogenesis of Multiple Myeloma. Cancers (Basel) 2019;11:1738.

11. Raimondi L, De Luca A, Morelli E, et al. MicroRNAs: Novel Crossroads between Myeloma Cells and the Bone Marrow Microenvironment. Biomed Res Int 2016;2016:6504593.

12. Abdi J, Qiu L, Chang H. Micro-RNAs, New performers in multiple myeloma bone marrow microenvironment. Biomark Res 2014;2:10.

13. Ma J, Liu S, Wang Y. MicroRNA-21 and multiple myeloma: small molecule and big function. Med Oncol 2014;31:94.

14. Yu T, Du C, Ma X, et al. Polycomb-like Protein 3 Induces Proliferation and Drug Resistance in Multiple Myeloma and Is Regulated by miRNA-15a. Mol Cancer Res 2020;18:1063-73.

15. Li Y, Gao S, Xue W, et al. mir-19a-3p Functions as an Oncogene by Regulating FBXO32 Expression in Multiple Myeloma. Balkan Med J 2021;38:43-9..

16. Xu T, Zeng Y, Shi L, et al. Targeting NEK2 impairs oncogenesis and radioresistance via inhibiting the Wnt1/ $\beta$-catenin signaling pathway in cervical cancer. J Exp Clin Cancer Res 2020;39:183.

17. Li B, Guo X, Li N, et al. WNT1, a target of miR-34a, promotes cervical squamous cell carcinoma proliferation and invasion by induction of an E-P cadherin switch via the $\mathrm{WNT} / \beta$-catenin pathway. Cell Oncol (Dordr) 2020;43:489-503.

18. Xu Z, Ran J, Gong K, et al. LncRNA SUMO1P3 regulates the invasion, migration and cell cycle of gastric cancer cells through Wnt/ $\beta$-catenin signaling pathway. J Recept Signal Transduct Res 2020;1-8.

19. Wang $\mathrm{H}$, Gong $\mathrm{Y}$, Liang L, et al. Lycorine targets multiple myeloma stem cell-like cells by inhibition of $\mathrm{Wnt} / \beta$-catenin pathway. Br J Haematol 2020;189:1151-64.

20. Wu X, Liu Y, Zhang E, et al. Dihydroartemisinin Modulates Apoptosis and Autophagy in Multiple Myeloma through the P38/MAPK and Wnt/ $\beta$-Catenin Signaling Pathways. Oxid Med Cell Longev 2020;2020:6096391. 
21. Wu Z, Yu B, Jiang L. MiR-212-3p mediates apoptosis and invasion of esophageal squamous cell carcinoma through inhibition of the $\mathrm{Wnt} / \beta$-catenin signaling pathway by targeting SOX4. J Thorac Dis 2020;12:4357-67.

22. Chang SH, Luo S, Thomas TS, et al. Obesity and the Transformation of Monoclonal Gammopathy of Undetermined Significance to Multiple Myeloma: A Population-Based Cohort Study. J Natl Cancer Inst 2016;109:djw264.

23. Zhong Y, Liu Z, Li D, et al. Identification and Validation of a Potential Prognostic 7-lncRNA Signature for Predicting Survival in Patients with Multiple Myeloma. Biomed Res Int 2020;2020:3813546.

24. Chen F, Wang X, Fu S, et al. Effect of the Up-Regulation of Circular RNA Hsa_circ_0069767 Derived from C-KIT on the Biological Behavior of Multiple Myeloma Cells. Cancer Manag Res 2020;12:11321-31.

25. Pan Y, Jin K, Xie X, et al. MicroRNA-19a-3p inhibits

Cite this article as: Wei Z, Wang W, Li Q, Du L, He X. The microRNA miR-19a-3p suppresses cell growth, migration, and invasion in multiple myeloma via the $\mathrm{Wnt} / \beta$-catenin pathway. Transl Cancer Res 2021;10(2):1053-1064. doi: 10.21037/tcr-20-3490 the cellular proliferation and invasion of non-small cell lung cancer by downregulating UBAP2L. Exp Ther Med 2020;20:2252-61.

26. Su YF, Zang YF, Wang YH, et al. MiR-19-3p Induces Tumor Cell Apoptosis via Targeting FAS in Rectal Cancer Cells. Technol Cancer Res Treat 2020;19:1533033820917978.

27. Zhan T, Rindtorff N, Boutros M. Wnt signaling in cancer. Oncogene 2017;36:1461-73.

28. van Loon K, Huijbers EJM, Griffioen AW. Secreted frizzled-related protein 2: a key player in noncanonical Wnt signaling and tumor angiogenesis. Cancer Metastasis Rev 2021;40:191-203.

29. Jung YS, Park JI. Wnt signaling in cancer: therapeutic targeting of Wnt signaling beyond $\beta$-catenin and the destruction complex. Exp Mol Med 2020;52:183-91.

(English Language Editor: J. Teoh) 